Cahiers de recherches médiévales

Journal of medieval studies

$8 \mid 2001$

La protection spirituelle au Moyen Âge

\title{
Droit canonique et protection des « cercles de paix 》)
}

\section{Thomas Gergen}

\section{(2) OpenEdition \\ 1 Journals}

Édition électronique

URL : https://journals.openedition.org/crm/404

DOI : $10.4000 / \mathrm{crm} .404$

ISSN : 1955-2424

Éditeur

Honoré Champion

\section{Édition imprimée}

Date de publication : 15 janvier 2001

Pagination : 135-142

ISSN : $1272-9752$

\section{Référence électronique}

Thomas Gergen, «Droit canonique et protection des « cercles de paix » , Cahiers de recherches médiévales [En ligne], 8 | 2001, mis en ligne le 13 mars 2008, consulté le 15 décembre 2022. URL : http://journals.openedition.org/crm/404 ; DOl : https://doi.org/10.4000/crm.404

Ce document a été généré automatiquement le 15 décembre 2022.

Tous droits réservés 


\title{
Droit canonique et protection des « cercles de paix »
}

\author{
Thomas Gergen
}

1 Quand le médiéviste pense au vocabulaire de la protection spirituelle, ce sont la Paix et la Trêve de Dieu qui vont inéluctablement lui venir à l'esprit et, en particulier, les différents termes des "cercles de paix » et des lieux sacrés, créés aux $\mathrm{X}^{\mathrm{e}}$-XIII ${ }^{\mathrm{e}}$ siècles pour limiter et encadrer les guerres privées. Ici, deux sources juridiques sont notamment à prendre en considération, d'une part les comptes rendus des conciles de Paix, d'autre part les compilations du droit coutumier, puisque droit canonique et droit laïc s'interpénètrent à l'époque en question.

2 Le mouvement de la Paix est né en Auvergne et Velay (Le Puy, vers 975) et en Aquitaine (Charroux, 989), à l'époque de la mutation dite féodale autour de l'an mil ${ }^{1}$. Les conciles de Paix tenus à partir de la deuxième moitié $\mathrm{du} \mathrm{X}^{\mathrm{e}}$ siècle se sont répartis dans toute l'Europe occidentale et ont établi un cadre juridique pour la protection des lieux sacrés ${ }^{2}$. Même si l'intérêt principal, dans le sillage de la tradition carolingienne, était la protection des pauperes et des clercs ainsi que de leurs biens, les lieux sacrés recevaient une mention spéciale dans les canons promulgués aux conciles et aux assemblées de Paix.

3 Pour rendre compte des " cercles de paix », de multiples expressions apparaissent dans les textes. La lecture des différences de vocabulaire dans l'Occident du $\mathrm{XI}^{\mathrm{e}}$ siècle nous donnera l'occasion d'étudier la fonction de mots dont l'emploi synonymique pose parfois des problèmes d'interprétation.

4 C'est tout d'abord l'ecclesia qui est l'objet d'une protection. Quiconque viole une église sainte ou en arrache quelque chose par la force sera puni par un anathème, à moins qu'il ne soit venu à satisfaction (restitution des biens, dommages et intérêts), énoncent les pères du concile de Charroux en 989: Anathema infractoribus ecclesiarum. Si quis ecclesiam sanctam infregerit, aut aliquid exinde per vim abstraxerit, nisi ad satis confugerit factum, anathema sit ${ }^{3}$. Cette protection est encore reprise au concile de Poitiers en 1011-1014 4 et l'ecclesia figure également dans la liste des endroits protégés proposés par le concile du Puy de $994^{5}$. Dès lors, les églises bénéficièrent d'une grande protection 
contre les tentatives d'effraction. L'ecclesia était le lieu d'immunité par excellence, où les guerres privées n'étaient pas permises. De surcroît, les églises représentaient un lieu d'asile en faveur des inermes, les non-armés, aussi bien clercs que laïcs de la société de l'époque.

Le concile de la Paix de Cologne tenu en $1083^{6}$ reflète magistralement ce zeitgeist, car l'hommage et le respect envers Dieu devait amener en retour la protection aux églises et aux cimetières, de sorte qu'un brigand ou un voleur qui s'y était réfugié ne pouvait être ni tué ni capturé. Il était cependant contraint de rester là jusqu'au moment où il abandonnerait les lieux pour cause de faim. Celui qui osait lui apporter des armes, de la nourriture, ou l'aidait à s'enfuir, devait subir la même sanction que le coupable ${ }^{7}$.

Nous trouvons également des indications plus précises que la notion d'ecclesia et qui décrivent des zones de paix plus complexes. A la fin $d u \mathrm{X}^{\mathrm{e}}$ siècle et au début du $\mathrm{XI}^{\mathrm{e}}$ siècle, les aîtres (atria) furent placés explicitement sous la protection de la Paix de Dieu, réactualisant ainsi le droit d'asile et l'immunité des cimetières ${ }^{8}$. La charte du concile du Puy-Saint-Paulien de l'an 994 n'évoque pas le mot d'atrium, mais elle mentionne l'ecclesia et ses alentours, en punissant tous ceux qui, aussi bien dans l'aître qu'en dehors de l'église ou dans son enceinte fortifiée, se seraient sciemment emparés de chevaux, de bœufs, de vaches, d'ânes et d'ânesses, ainsi que de leurs fardeaux, ou de moutons, de chèvres, de porcs, ou ne les tuerait, si ce n'est lorsqu'ils recevraient en chemin de la nourriture pour eux-mêmes ou pour leurs gens 9 .

Le terme d'atrium est par ailleurs évoqué par le concile de Verdun-sur-le-Doubs, tenu en 1016 : atria ecclesia $e^{10}$. Il semble équivalent de la cellaria dont il est question lors du concile d'Anse en $994^{11}$ ou de celui de Beauvais, plus tard, en $1023^{12}$. En 1095, le concile de Clermont a, de plus, étendu l'immunité et l'asile aux croix des chemins ${ }^{13}$.

Des recherches récentes ont porté sur les cercles protégés dans le Midi de la France et surtout sur la Catalogne, région où se développèrent très tôt les convenientiae, pactes de paix négociés pour que deux partis règlent une question conflictuelle et puissent se réconcilier ${ }^{14}$. La Paix fut réaffirmée en Catalogne lorsque, le 16 mai 1027, à Toulouges près d'Elne dans le Roussillon, des évêques ainsi que d'autres ecclésiastiques et laïcs se réunirent pour le premier concile de Paix sur le territoire catalan et jurèrent un pactum sive treugam, expression de protection qui devint dorénavant fréquente. C'est plus particulièrement la Paix du dimanche, menacée, qui tenait à cœur aux participants de cette assemblée. Les ecclésiastiques, les hommes et femmes non armés ainsi que les églises et les maisons qui se trouvaient à l'intérieur d'un cercle de trente pas de rayon autour, furent placés sous une protection de Paix particulière.

Dans la société catalane contemporaine, la notion de sacraria (latin pour «choses sacrées »; en catalan, la "sagrera », les « sagreres »; en français, la «sacrée ») était présente, une institution que le philologue catalan Joan Coromines a défini comme le quartier formé par l'église et ses dépendances: «el barri format par l'església i les serves dependènciès $»^{15}$. Antoni $\mathrm{M}$. Alcòver va plus loin, en mettant en jeu la possibilité d'asile et de refuge en faveur des délinquants : «l'església i les seves dependències, que constituïen el sagrat o lloc d'asil per als delinqüents ${ }^{16}$.

Dans des recherches plus précises, Pierre Bonnassie démontre que la sacraria était le rayon de trente pas, protégé car sacré, qui entourait l'église et jouissait de la même protection qu'elle. À l'intérieur, il y avait des bâtiments, étroitement imbriqués qui s'appelaient eux-mêmes "sagreres ${ }^{17}$. À partir de la porte du temple et tout autour d'elle, on comptait trente pas, de manière à ce que, dans l'ensemble de ce cercle (in toto 
circuitu), les fugitifs jouissent d'une protection parce qu'ils se trouvaient en un lieu saint, in loco sancto ${ }^{18}$. Grâce à cette immunité, la sacraria relevait de la juridiction ecclésiastique et, par conséquent, du domaine protégé par les conciles, puis par le droit coutumier, en l'espèce les Usages de Barcelone ${ }^{19}$. L'évêque avait le seul pouvoir de juger des méfaits qui y étaient commis, de même qu'il jugeait seul les crimes ou délits perpétrés à l'intérieur de l'édifice ecclésial ${ }^{20}$.

11 Pour notre étude du vocabulaire de la protection, il est dès lors intéressant de suivre la diffusion et la fonction de la sacraria qui paraît connaître une évolution parallèle à d'autres expressions, les « trente pas » et le cimi(n)nterium.

Certes, le terme de sacraria (sagrera) était connu depuis longtemps. Le premier exemple de son utilisation se rapporte à la donation d'un alleu à la cathédrale Sainte Eulàlia de Brouillà, située au sud-ouest d'Elne, en l'an 976 : cum ipsos sacrarios qui sunt prope sancto Iohanne $^{21}$. Aymat Catafau envisage, à juste titre, la naissance des celleres dans un contexte de crise et de violences, ce qu'indique l'intitulé d'un des chapitres de sa thèse, "Le Roussillon, le Conflent et le contexte de crise et de violence ${ }^{22}$. Du fait de la multiplication des conflits internes aux groupes aristocratiques qui opposaient entre eux les représentants les plus éminents de cette couche sociale et leurs hommes d'armes, les synodes de la Paix et Trêve de Dieu, tels que ceux de Toulouges en 1027 et 1064, apparurent comme la seule tentative de régulation des violences ${ }^{23}$. La sauveté des trente pas est devenue une institution ferme de la Paix, voire une transposition visible et pratique ${ }^{24}$, car l'urbanisme mettait en œuvre ce programme politique. Les cercles de paix contribuèrent donc largement au développement de l'art roman et furent à l'origine de nombreux villages catalans ${ }^{25}$.

13 La plupart du temps les Usages de Barcelone évoquent les «trente pas » et traduisent fidèlement des mots des conciles wisigothiques. L'article 133 qui, à plusieurs reprises, utilise ce terme des "trente pas ${ }^{26}$ rappelle le canon $10 \mathrm{du}$ XII ${ }^{\mathrm{e}}$ concile de Tolède en l'an 681, concernant le droit d'asile. Les évêques, avec l'approbation du roi wisigothique Ervige, avaient borné cet asile ecclésiastique au cercle des «trente pas » entourant les églises: Sed in hoc XXX passuum numero absque domorum extranearum receptaculis progrediendi aditum obtinebunt et in quibus triginta passibus unuscuiusque ecclesiae in toto circuito reverentia defendetur ${ }^{27}$.

14 Cependant, le mot sacraria figure également dans les Usages; on peut alors se poser la question de savoir pourquoi les Usages n'ont adopté que ponctuellement l'expression de sacraria bien que celle-ci ait eu le même sens que celle des «trente pas ${ }^{28}$.

15 L'article 173 préconise le respect des cimetières, de la sacrée ainsi que celui des trente pas: ullos homo ecclesiam non infrigat, eque cimiteria sacraria triginta passuum ecclesiasticorum in circuitu uniuscuiusque ecclesie ${ }^{29}$. Cet article a hérité d'une chaîne de synonymes par le biais du concile de Toulouges, puisque nous trouvons ici une triple synonymie, à savoir la sacrée, le cimetière et les trente pas.

16 Le concile de Toulouges de 1064-1066 est en fait le seul concile de Paix catalan du $\mathrm{XI}^{\mathrm{e}}$ siècle qui emploie la notion de sacraria. Cependant, les trente pas sont mentionnés immédiatement après et le nom de ciminterium précède ces deux dénominations. L'emploi simultané de la "sacrée » et des «trente pas " nous amène donc à parler d'abord d'une accumulation des deux synonymes. Puisque l'on faisait déjà l'amalgame entre cimetière et sacrée dès la fin du $\mathrm{XI}^{\mathrm{e}}$ siècle, on peut avancer l'idée d'une triple synonymie, exprimée dans le canon significatif du concile de Toulouges évoqué cidessus $^{30}$. Il s'agit du seul cas où la richesse du vocabulaire utilisé fut l'objet d'une 
réception dans les Usages de Barcelone ${ }^{31}$. Ceux-ci, en général, ne réemploient que les "trente pas ", réception qui se fonde sur la tradition des conciles de Paix catalans. Ces derniers ont suivi la tradition du concile de Tolède et entérinent la notion des trente pas ecclésiastiques. Le concile de Toulouges de 1027 sanctionne les briseurs de la zone des trente pas $^{32}$, de même celui de Vic de $1033^{33}$. Lors du concile de Barcelone il a été prévu contre l'atteinte aux trente pas une sanction de 600 sous, sanction traditionnelle dans le droit romain ${ }^{34}$. A Vic-Gérone en 1064, il est deux fois question des trente pas ecclésiastiques alors que la sacraria n'apparait pas ${ }^{35}$.

17 À l'opposé des conciles français mentionnés plus haut, le concile de Narbonne (1054) entre dans la tradition des conciles catalans, car on y a aussi utilisé le cercle des trente pas pour décrire la zone de paix ${ }^{36}$. En 1131, Raimon Berenguer III, en collaboration avec l'archevêque de Tarragone, Oleguer, instaura la protection des "trente pas » et des églises qui n'étaient pas fortifiées. L'assemblée stipula encore une amende de 600 sous pour le cas des briseurs de l'immunité de la sacrée.

18 Les constitutions de Paix et Trêve de 1173, promulguées pour le Roussillon par Alfonse $\mathrm{I}^{\mathrm{er}}$, roi d'Aragon, sont la première initiative publique de défense des églises, cimetières et de la sacrée, plus d'un siècle après le dernier synode de Toulouges. Dans l'intervalle, aucune prise de position des comtes n'était venue défendre les espaces de paix. L'incapacité des comtes à empêcher les troubles des $\mathrm{XI}^{\mathrm{e}}$ et XII ${ }^{\mathrm{e}}$ siècles, pire, le rôle actif qu'ils ont pris maintes fois dans les guerres privées, les rapines et les actes sacrilèges, expliquent clairement cette incurie. En 1173, le roi fit signer la Paix à un groupe représentatif des familles nobles les plus puissantes du Roussillon. Ce n'est peut-être pas un hasard si nous trouvons, en de nombreux endroits de la marche catalane, la cellaria répandue justement à cette époque ${ }^{37}$.

Dans la Constitution de Jaume $\mathrm{I}^{\mathrm{er}}$ en 1228, le mot sacraria a été utilisé à côté de la notion du cimenteri. Apparemment le souverain voulait employer un mot plus populaire pour qualifier le domaine de la Paix. Du reste, à cette époque la synonymie des notions de ciminteri et de sagrera était complètement achevée. Il est frappant qu'aucune allusion ne soit faite aux trente pas. Le paragraphe le plus éloquent stipule, en langue catalane cette fois-ci ${ }^{38}$. La constitution de Paix et Trêve de Jaume $1^{\text {er }}$ est considérée comme le dernier moment important du mouvement de la Paix en Catalogne. Elle figure une dernière fois le mouvement tel qu'il s'est développé jusqu'au XIII ${ }^{\mathrm{e}}$ siècle $^{39}$.

Mais tournons-nous également vers la toponymie. Bien que des localités soient indiquées comme s'appelant «trentepasses", le terme catalan "sagrera " est sans équivoque plus populaire pour les appellations locales. Nous retrouverons de telles indications à Montornès, Lliça d'Amunt, Palau de Plegamans, Santa Eulàlia de Ronçana, comme hameau (village) de la commune de Martorelles (Vallès). Un document de Sainte-Marie de Coustouges mentionne, en 988, les «sagrers » non pas nommément, mais en les définissant comme des maisons situées autour de l'église : ecclesia sancte Marie... cum suo cimiterio et cum ipsas domos que sunt circa ipsam ecclesiam.

21 Dans une charte de donation de Saint-Estève de Riuferrer de 993, les "trente pas » entrent de nouveau en jeu : donamus in circuitu ecclesiarum cimiteria secundum canonicam autoritatem in circuitu ecclesie Sancti Stephani cimiterium triginta passuum ${ }^{40}$.

La teneur d'une notice judiciaire (querimonia) nous renseigne finalement sur la signification pratique de la sacrée. Elle concerne, en effet, la violation d'un secteur protégé au temps de la trêve. Les milites d'Artal $1^{\mathrm{er}}$ avaient pillé et incendié les sacrées du Pallars. Nous citons les passages les plus importants de la traduction empruntée à 
Pierre Bonnassie ${ }^{41}$ : «Il rompit la Trêve de Dieu, à Mala, à Enseu ; à Torella, pendant la Trêve de Dieu, il détruisit (fregit, brisa) les sacrarios; à Agramunt, pendant la trêve de Dieu, il brisa les sacrarios ; il rompit la Trêve de Dieu et brisa les sacrarios à Segur... ». On peut trouver d'autres exemples de la présence de la sacrée dans les actes de vente, de donation, des testaments, des plaintes, ainsi que des documents qui, en raison de leur utilisation dans la sphère privée, possèdent un caractère plutôt populaire ${ }^{42}$.

L'expression de sacraria est vraisemblablement propre à la Catalogne, où elle fait fonction de désignation spécifique et régionale pour les espaces protégés autour des églises, c'est-à-dire les « trente pas » apparaissant dans presque toutes les catégories de sources. Son usage s'inscrit dans la tradition des conciles de la péninsule ibérique, en particulier ceux de Tolède. En France, ce sont les notions de cellaria, atrium, ecclesia, qui se sont implantées.

Depuis le concile de Tolède de 681, les «trente pas » expriment essentiellement la notion de zone protégée, de même - mais très rarement - que dans les documents privés. Dans ce cas, la sacrée est un terme beaucoup plus familier, un terme juridique plus populaire, car vis-à-vis des "trente pas» elle prédomine d'un point de vue toponymique et se rencontre pour des usages privés et écrits. Cette institution, qui a donné son nom à bon nombre de sites, a créé une certaine conscience dans la société qui devait dorénavant respecter l'immunité de ces « cercles de paix ». En même temps, les guerres privées furent encadrées et limitées tant en durée qu'en dimension.

En revanche, les «trente pas » apparaissent dans les textes des conciles et se trouvent ainsi dans la tradition juridique de l'Église, c'est-à-dire la tradition savante. Ils finissent par figurer dans les Usages de Barcelone où, en tant que terme important du mouvement pacifiste, ils indiquent exactement la zone protégée sur laquelle s'étendait la Treuga $D e^{43}$.

\section{NOTES}

1. J.-P. Poly et E. Bournazel, La mutation féodale ( $\mathrm{X}^{e}-\mathrm{XII}{ }^{e}$ siècles), Paris, PUF, 1991, pp. 222-223 ; C. Lauranson-Rosaz, L'Auvergne et ses marges (Velay, Gévaudan) du VII au XI siècle: la fin du monde antique?, Le Puy-en-Velay, 1987, pp. 410-411 ; R. Favreau, « Le concile de Charroux (989) et la Paix de Dieu ", Bulletin de la Société des Antiquaires de l'Ouest, 3, 1989, 5e série, pp. 213-219.

2. H.-W. Goetz, «La Paix de Dieu en France autour de l'an mil : fondements et objectifs, diffusion et participants ", Le roi de France et son royaume autour de l'an mil, dir. M. Parisse et X. Barral I Altet, Paris, Picard, 1992, pp. 131-145 ; T. Gergen, « Texttradition der Usatges de Barcelona am Beispiel von pau i treva und den XXX passes (sagreres) der katalanischen Friedenskonzilien », Mélanges D. Briesemeister, éd. S. Grosse et A. Schönberger, Berlin, 1999, pp. 267-273.

3. J.-D. Mansi, Sacrorum conciliorum nova et amplissima collectio, Florence-Venise, 1759-1798, réed. Paris, 1899-1927, XIX, col. 89-90 ; J.-P. Brunterc'h, Le Moyen Âge ( $V^{e}-X I^{e}$ siècles), t. 1, Paris, Fayard, 1994, p. 381.

4. L. Huberti, Studien zur Rechtsgeschichte der Gottesfrieden und Landfrieden in Frankreich, Ansbach, 1892, p. 137. 
5. Sources d'histoire médiévale, IX ${ }^{e}-m i l i e u ~ d u X I V{ }^{e}$ siècles, dir. G. Brunel et E. Lalou, Paris, Larousse, 1997, pp. 130-131.

6. K. Kroeschell, Deutsche Rechtsgeschichte, t. I, $11^{\text {e }}$ éd., Wiesbaden-Opladen, 1999, pp. 188-194.

7. In aecclesiis vero et aecclesiarum cimiteriis honor et reverentia Deo prebeatur, ut si illuc confugerit raptor vel fur, minime interficiatur vel capiatur, sed tam diu inibi obsideatur, donec fame urguente ad deditionem cogatur. Si aliqua persona reo arma, victum vel fugam moliri presumpserit, aequalem poenam sicut reus susustnebit.Voir MGH, Constitutiones, I, no. 424.

8. C. Treffort, "Consécration de cimetière et contrôle épiscopal des lieux d'inhumation au $\mathrm{X}^{\mathrm{e}}$ siècle ", Le sacré et son inscription dans l'espace à Byzance et en Occident, dir. M. Kaplan, Paris, Publications de La Sorbonne, 2001, pp. 285-296; id., L'église carolingienne et la mort. Christianisme, rites funéraires et pratiques commémoratives, Lyon, 1998, pp. 141-143.

9. ...extra aecclesiam quam in firmamento castellie se sciente nisi episcopi propter eorum censum predam in istis comitatibus neque in istis episcopatibus homo non faciat de equis publicis, de bovibus, de vaccis, de asinis vel de asinas, vel de fascibus quos ipsi portant, neque de ovibus, vel de capris, neque de porcis, neque occidat, nec per conductum suum vel suorum in itinere positus accipiat victum... ;cf. C. Lauranson-Rosaz, op. cit., p. 421.

10. Audite christiani convenentiam pacis. Ecclesiam nullo mdo infringam. Atria ecclesiae non infringam, nisi propter ipsum malefactorem qui hanc pacem infregerit : et si pisa atria infringero, nihil inde traham nisi ipsum malefactorem aut eius guarnimentum; cf. Bonnaud-Delamare, «Les institutions de paix dans la province ecclesiastique de Reims au XI ${ }^{\mathrm{e}}$ siècle », Bulletin philologique et historique,57, 1955-1956, pp. 148-149.

11. ...ecclesiis cum domibus et cellariis ad eundem locum pertinentibus... ; cf. L. Huberti, pp. 1-42.

12. Sacramentum pacis. Ecclesiam nullo modo infringam. Cellaria in circuitu ecclesiae causa salvamenti eiusdem non infringam, nisi propter illum malefactorem, qui hanc pacem infregerit, aut propter factum homicidium aut propter comprehensionem hominis aut caballi. Et si propter has res eadem cellaria infregero, nihil aliud ex eis traham nisi illum malefactorem aut eius warnimentum me sciente; L. Huberti, pp. 165-167.

13. XXIX. Ut liberi sint qui ad crucem confugiunt. Si quis ad aliquam crucem in via persequentibus inimicis confugerit, liber ac si in ipsa ecclesia permaneat. - XXX. De iis, qui ad crucem vel ecclesiam confugiunt. Quod si quis pro securitate ecclesiae vel praedictae crucis aliquod crimen peregerit, et ad ecclesiam vel crucem confugerit, accepta securitate vitae et membrorum, reddatur iustitiae. Cf. J.-D. Mansi, op. cit., XX, p. 818 ; L. Huberti, op. cit., p. 408.

14. O. Guillot, Y. Sassier, A. Rigaudière, Pouvoirs et institutions dans la France médiévale, t. I : des origines à l'époque féodale, Paris, Armand Colin, 1999, 3 e éd., pp. 204-205.

15. J. Coromines, Diccionari etimologic i complementari de la llengua catalana, vol. 7, Barcelone, 1996, p. 593.

16. A.M. Alcover, Diccionari català-Valencià-Baleàar, vol. 9, Palma de Mallorca, 1977, p. 671.

17. P. Bonnassie, «Les sagreres catalanes : la concentration de l'habitat dans le cercle de paix des églises (XI ${ }^{\mathrm{e}}$ siècle) », L'environnement des églises et la topographie religieuse des campagnes médiévales, Actes du III Congrès International d'Archéologie Médiévale, Aix-en-Provence, 28-30 sept. 1989, dir. M. Fixot et E. Zadora-Rio, Paris, 1994, p. 68 ; T. Bisson, Tormented voices : power, crisis and humanity in rural Catalonia (1140-1200), Cambridge (Massachussets)-Londres, 1998, p. 124.

18. M. Riu et P. Valdepenas, «El espacio ecclesiastico y la formacionn de las parroquias en la Cataluna de los siglos IX al XII », Fixot \& Zadora-Rio, op. cit., p. 58.

19. P. Bonnassie, art. cit., p. 71 ; K. Kennelly, « Sobre la paz de Dios y la sagrera en el condado de Barcelona (1030-1130) », Anuario de Estudios Medievales, 5, 1968, pp. 107-136.

20. P. Bonnassie, art. cit., p. 69.

21. A. Catafau, Les celleres et la naissance du village en Rousillon (Xe-XVe siècles), Perpignan, 1998,

p. 61 et pp. 232-234.

22. Id., pp. 39-55. 
23. Id., pp. 40-41.

24. D. Barthélémy, L'an mil et la paix de Dieu. La France chrétienne et féodale, 980-1060, Paris, Fayard, 1999, pp. 512-513 ; K. Kennelly, art. cit., p. 136.

25. P. Bonnassie, art. cit., p. 75.

26. Ille autem homo qui aliter ecclesiam invaserit aut ea que in circuitu eius sunt usque ad XXXa passusirruperit, summam sexcentorum solidorum pro sacrilegii composicione emendet, et tamdiu excomunicetur quousque digne satisfaciat. Cf. Valls I Taberner, Édicion y comentarios de los Usatges de Barcelona, Barcelone, 1994, pp. 111-112.

27. Pour les références précises, voir P. Bonassie, art. cit., p. 72 et p. 78.

28. Ce n'est pas le lieu de faire une ébauche de l'origine et du contenu des Usages ainsi que de leurs liens avec les mouvements de paix dans l'Europe occidentale du Moyen Âge. Cf. T. Gergen, « Texttradition... », op. cit., Berlin, 1999, p. 259.

29. Valls I Taberner, op. cit., pp. 132-135.

30. Constituerunt, namque, predicti pontifices, cum consensu ceterorum nobilium, ut in comitatu Russilionensi vel Confluentano, vel Valle Spirensi, ullos homo ecclesie non infringat, neque cimiteria vel sacraria, XXXa. Passuum ecclesiasticorum in circuitu unuscuiusque ecclesie, nisi episcopus propter hominem excommunicatum aut propter suum censum. Si vero aliquis homo aliter infregerit ecclesiam vel spacium XXXa. Passuum ipsius ecclesie in predicto episcopatu Elenensi, quicquid ei comiserit, emendet, ut iustum fuerit, ipsi homini cui iniuriam fecerit, et ecclesie in qua fecerit satisfaciat legaliter, et insuper composicionem sacrilegii Elenensi componat episcopo. Cf. G. Gonzalvo I Bou, Les Constitutions de Pai $i$ Treva de Catalunya (segles XI-XIII), Bercelone, 1994, pp. 30-31.

31. En revanche, l'expression de sacraria ne se trouve pas mentionnée dans la version catalane. Restent alors seulement les notions de cimiteria et de XXX passes, «ni cimiteris, trenta passos ecclesiàstics ». Voir T. Gergen, « Texttradition... », op. cit., p. 269.

32. ...neque aliquis auderet ecclesiam vel domos in circuitu positas a XXX passibus violare aut assallire..., cf. G. Gonsalvo I Bou, op. cit., p. 4.

33. Neque mansiones que in circuitu ecclesie sunt aut erunt usque ad XXX passi. Ille autem homo qui aliter ecclesiam invaserit aut ea que in circuitu eius sunt usque ad XXX passus irrupit, sacrilegio compositionem emendet. Cf. G. Gonsalvo I Bou, op. cit., p. 9.

34. Constitucione namque predictorum episcoporum et principum sancitum est ut ab ista die et deinceps, nullus utriusque sexus homo ecclesiam nec mansiones que in circuitu ecclesie sunt aut erunt, usque ad XXX passus, non infringat aut invaat...

Ille autem homo qui ecclesiam aliter invaserit, aut ea que in circuitu eius sunt usque ad XXX passus irruperit, summam DC solidorum pro sacrilegii composicione emendet. Cf. G. Gonsalvo I Bou, op. cit., pp. 14-15.

35. Ut ab ista hora die et deinceps, ullus homo ecclesiam non infringat neque spacium neque cimiterium neque mansiones, que in circuitu ecclesie sunt aut erunt usque ad XXX ecclesiasticos passus...

Ille autem homo qui aliter ecclesias invaserit, aut aliis exceptis supradictis malefactoribus aliquid ibi malefecerit, aut ea que in circuitu eiusdem ecclesie sunt usque ad XXX ecclesiasticos passus irruperit, sacrilegii composicionem emendet pretate Sedi et querelanti malefacta in duplo componat. Cf. G. Gonsalvo I Bou, op. cit., pp. 22-23.

36. L. Huberti, op. cit., p. 319.

37. A. Catafau, op. cit., p. 54.

38. «Encara los siminteris e les sacreres vel sacraria, de qualque esgleya entorne d'aquela establides, negu no asaig esvair ne trencar-ne neguna cosa d'aquen trer. Als trencadors emperò d'aquest establiment, pena de sacrilegi los sia posada, e per lo bisbe d'aquel bisbat sein destrets de satisfer lo dan en doble a quel a qui serà lo dan donat ». Cf. G. Gonsalvo I Bou, op. cit., p. 37.

39. G. Gonsalvo I Bou, La Pau i Treva a Catalunya: origen de les Corts Catalanes, Barcelone, 1986, pp. 114-115.

40. Voir P. Bonnassie, art. cit., p. 72. 
41. P. Bonnassie, art. cit., pp. 74-75.

42. T. Gergen, « Texttradition... », op. cit., pp. 272-273.

43. Il s'agit là d'un exemple particulièrement pertinent de la réception juridique et lexicale des conciles ecclésiastiques dans le droit coutumier territorial ; cf. T. Gergen, « Paix éternelle et paix temporelle : tradition de la Paix et de la Trêve de Dieu dans les compilations du droit coutumier territorial », Cahiers de civilisation médiévale, 2002 (sous presse).

\section{AUTEUR}

\section{THOMAS GERGEN}

Institut d'histoire du droit et de droit civil, Université de Sarrebrück/CESCM, Université de Poitiers 